\title{
STRAFRECHTLICHE PRODUKTHAFTUNG
}

Ürün Sorumluluğunun Cezai Boyutu

\section{Henning ROSENAU}

\section{ÖZET}

Ürün sorumluluğu kavramının cezai boyutu, Almanyada ilk defa yetmişli yılların başında Schmidt-Salzer isminde Honneverli bir avukat tarafindan keşfedilmiştir. Bunun hemen akabinde yargı ve doktrin konuyu ele almaya başlamış ve ürün sorumluluğunun cezai boyutu hakkında bir takım sistematik yapılar oluşturulmak için çalışmalara girişilmiştir. Ürün sorumluluğunun cezai boyutu konusunda iki ana sorunla karşılaşmaktayız. Bunlardan birincisi illiyet bağının ispatıdır. Bu noktada genel illiyet bağ kavramından yararlanılmaktadır. Diğer sorun ise, şirketlerin yönetim kurulu üyelerinin de cezai sorumluluklarının bulunup bulunmadığı hususudur. Güven prensibinden kaynaklanan bir takım sınırlandırmalar söz konusu olmasına rağmen, günümüzde cezai ürün sorumluluğu, işletmelerde cezai compliance kapsamında karşımıza çıkan, deyim yerindeyse "kesin bir kılıç" görünümündedir.

Anahtar Sözcükler: Ürün sorumluluğu, genel illiyet bağı (isnadiyet), genel sorumluluk, güven prensibi, cezai sorumluluğun üstlenilmesi

\begin{abstract}
The criminal aspects of the concept of product liability was first discovered in Germany in the early 70's by a lawyer from Hanover named Schmidt-Salzer. Immediately after, the judiciary and the academia began to deal with the subject and works began to create systematic structures on the
\end{abstract}

* Prof. Dr., Augsburg. 
criminal aspects of product liability. We are facing two main problems with the criminal aspects of product liability. The former is the proving of causal connection. At this point, the concept of general causal connection is utilized. The latter is the question of whether the members of the companies' board of directors also bear criminal responsibility. Despite the existence of several limitations stemming from the principle of trust, today the criminal product liability seems to be a "sharp sword" as far as the context of criminal compliance of firms is concerned.

\section{KEY WORDS}

Product liability, general causal connection, general responsibility, criminal compliance

\section{A. Einführung}

Die Relevanz des Strafrechts in der Produkthaftung ist zunächst unterschätzt worden ${ }^{1}$. Dabei war es ein Strafverfahren über einen Produktfehler, das lange den Rekord in der deutschen Prozessgeschichte hält, längstes Strafverfahren gewesen zu sein: das Contergan-Verfahren mit einer $2 \frac{1}{2}$-jährigen Hauptverhandlung und einem Ermittlungsverfahren von 6 $1 / 2$ Jahren hatte sich über 9 Jahre erstreckt, als es 1971 vom Landgericht Aachen wegen dieser langen Verfahrensdauer gem. $§ 153$ Abs. 2 StPO eingestellt wurde $^{2}$. In diesem Verfahren war es um die Strafbarkeit des Arzneimittelherstellers Grünenthal gegangen, dessen Medikament mit dem Wirkstoff Thalidomid nach der Einnahme durch Mütter Missbildungen bei den Neugeborenen bewirkte. Man könnte daher, aufgrund der hohen Anzahl der Geschädigten und der Beschuldigten, sowie der Länge des Verfahrens sagen, es sei der Vorzeige-Fall der strafrechtlichen Produkthaftung ${ }^{3}$. Die Konsequenz war in Deutschland die Entwicklung eines Arzneimittelgesetzes, um die Arzneimittelsicherheit zu gewährleisten.

Ein ähnlich breites Echo in den Massenmedien erregte das Verfahren um das ICE-Unglück bei Eschede im Jahre $1998^{4}$, bei dem ein geplatzter

1 Vgl. Schmidt-Salzer, Strafrechtliche Produktverantwortung, Das Lederspray-Urteil des BGH, NJW 1990, S. 2966 (Fn. 14); Kuhlen, Fragen einer strafrechtlichen Produkthaftung, Heidelberg 1989, S. 6 f., der die erste Monographie veröffentlichte; sowie Eidam, Unternehmen und Strafe, 3. Aufl. Köln 2008, Rn. 2398 ff.

2 Gem. § 153 Abs. 2 StPO, LG Aachen, JZ 1971, 507, 519 f.

${ }^{3}$ Momsen/Grützner/Voigtel, Wirtschaftsstrafrecht, München 2013, S. 1354, Rn. 69.

4 LG München II, in: ES Nr. IV, 28, S. 296 ff. 
Radreifen den Tod von 101 Menschen verursachte. Allerdings zeigt dieses Verfahren auch die Probleme strafrechtlicher Ahndung auf. Anders als im Zivilverfahren, wo wir auf der Grundlage europarechtlicher Richtlinien im ProdHaftG eine verschuldensunabhängige Gefährdungshaftung kennen, sind die beweisrechtlichen Anforderungen bei strafrechtlichen Verurteilungen sehr hoch. Ob die beiden angeklagten Ingenieure des Radherstellers wirklich keinen dem Stand von Wissenschaft und Technik entsprechenden Nachweis der Betriebssicherheit erbracht hatten, war trotz zahlreicher Sachverständiger nicht sicher nachzuweisen. Das Verfahren wurde schließlich nach $\S 153 \mathrm{a}$ Abs. 2 StPO eingestellt.

Bedenkt man den gravierenden Eingriff, den ein derartiges Strafverfahren für die Reputation eines Unternehmens bedeutet, und der die Arbeitsleistung und den Betriebsfrieden erheblich beeinträchtigt, so spürt man die Brisanz der strafbaren Produktverantwortung. Es scheint daher keineswegs überzogen, von der „Vierten Dimension des unternehmerischen Risikos" zu sprechen", einem Risiko, das sich nicht versichern lässt und bei dem sich auch nicht das Unternehmen schützend vor seine Mitarbeiter stellen kann.

\section{B. Die strafrechtliche Problematik}

Ein eigenständiges Institut einer strafrechtlichen Produktverantwortung ist dem deutschen Recht fremd ${ }^{6}$, eine Strafbarkeit kann sich folglich nur nach den Vorschriften des allgemeinen Strafrechts ergeben. Besondere Vorschriften finden sich lediglich in $\S \S 95,96$ AMG, $\S \S 58,59$ LFGB und $\S$ 27 ChemG - maßgeblich wird hierbei auf das Inverkehrbringen bestimmter Produkte abgestellt. Es handelt sich großteils um abstrakte Gefährdungsdelikte, eine tatsächliche Gefährdung ist nicht strafbegründend, wohl aber in einigen Fällen strafschärfend.

Das Strafrecht interessiert sich allerdings gerade wegen der möglichen Gefahren für Leib und Leben für die Produkthaftung ${ }^{7}$. In Betracht kommen primär die Straftatbestände, die die Rechtsgüter Leib und Leben schützen: vorsätzliche und fahrlässige Tötung ( $\S 212,222 \mathrm{StGB}$ ) sowie vorsätzliche

5 Schmidt-Salzer, Entscheidungssammlung, Produkthaftung, Band IV, München 1982, S. 161.

6 Schmidt-Salzer, Strafrechtliche Produktverantwortung, NJW 1988, S. 1937; Momsen/Grützner/Voigtel, Wirtschaftsstrafrecht, München 2013, S. 1334, Rn. 8.

7 Achenbach/Ransiek/Kuhlen, Wirtschaftsstrafrecht, 3. Aufl., Heidelberg 2012, S. 89, Rn. 17. 
und fahrlässige Körperverletzung ( $\S$ 223, 224, 229 StGB). Da freilich selbst bedingt vorsätzliches Handeln kaum beweisbar sein wird, geht es fast ausnahmslos um fahrlässiges Verhalten ${ }^{8}$.

Damit stellt sich für die Strafrechtswissenschaft die Aufgabe, wie sich die Verantwortung für Produktfehler in die Fahrlässigkeitsdogmatik einordnen lässt. Die Probleme beim Fahrlässigkeitsdelikt bestimmen auch die Probleme einer strafrechtlichen Produkthaftung: wie lässt sich ein kausales Verhalten nachweisen ${ }^{9}$ ? Wie ist die Frage des Verantwortlichen in einem arbeitsteilig organisierten Produktionsprozess zu beantworten ${ }^{10}$ ? Wonach ist der Sorgfaltsmaßstab zu bestimmen ${ }^{11}$ ? Inwieweit darf ein Mitarbeiter auf seine Kollegen vertrauen und wo liegt die Grenze dessen, was ihm zugemutet wird ${ }^{12}$ ?

\section{Strafrechtliche Produktverantwortung im Einzelnen}

\section{Nachweisbarkeit der Kausalität}

Am Anfang steht im Strafrecht die Frage der Kausalität des Verhaltens für den strafrechtlichen Erfolg. Im Gegensatz zum Privatrecht, wo nur sozial adäquate Verhaltensweisen ursächlich werden können, verlangt das Strafrecht nach der conditio sine qua non-Formel lediglich, dass das zu beurteilende Verhalten nicht hinweggedacht werden könnte, ohne dass der Erfolg in seiner konkreten Gestalt entfiele.

Diese Bedingungstheorie hat Bedeutung in zweifacher Hinsicht. Zum einen kann sich der Kreis der Verantwortlichen aus mehreren Personen zusammensetzen, wenn etwa der Letztverursacher, der fehlerhaft hantierende Arbeiter am Band wie ein Mitarbeiter in der oberen Betriebshierarchie, der etwa für die Überwachung zuständig ist, ein kausales Fehlverhalten an den Tag legt: beide sind alternativ kausal: Ihr Verhalten kann u.U. nicht hinweggedacht werden, ohne dass der Tod oder die Verletzung eines Menschen entfiele.

8 Im Lederspray (Erdal Rex)-Urteil ging es allerdings auch um § 224 StGB (gefährliche vorsätzliche Körperverletzung).

9 Vgl. Kuhlen (Fn. 1), S. 30 f.; Tiedemann, Fragen einer strafrechtlichen Produkthaftung, NJW 1990, S. 2051.

${ }^{10}$ Vgl. Schönke/Schröder/Sternberg-Lieben, § 15 Rn. 223a; Schmidt-Salzer (Fn. 1), NJW 1990, S. 2967.

${ }^{11}$ Vgl. Tiedemann (Fn. 9), NJW 1990, S. 2951, 2052; Kuhlen (Fn. 1), S. 30 f.; Schönke/Schröder/Sternberg-Lieben, § 15 Rn 223.

12 Vgl. Schmidt-Salzer, Produkthaftung, Band I: Strafrecht, 2. Aufl., Heidelberg 1988, S. 7. 
Auf der anderen Seite wird mit dieser Formel der Nachweis verlangt, dass gerade das fehlerhafte Produkt für den Schaden beim Verbraucher ursächlich war. Dies kann zu erheblichen Beweisschwierigkeiten führen ${ }^{13}$, wie sie z.B. im Contergan-Fall aufgetreten sind, wo die Wissenschaftler zwar eine Plausibilität für die Ursächlichkeit des Thaliodomids attestierten, aber ein naturwissenschaftlicher Nachweis umstritten blieb. Die Rechtsprechung behilft sich auf zweierlei Weise:

1. Es genügt zum einen, dass ein Produkt schadenserheblich war, und alle anderen denkbaren Alternativ-Ursachen ausgeschlossen werden können ${ }^{14}$. Der Wirkungsmechanismus im Einzelnen, der zum Schaden führte, muss nicht aufgedeckt werden ${ }^{15}$.

2. Des Weiteren könne der Richter über die generelle Kausalität, also der Frage, ob eine allgemeine Gesetzesaussage zutrifft, im Rahmen seines Beweiswürdigungsrechts urteilen. Er darf sich dabei zwar nicht über gesicherte wissenschaftliche Erkenntnisse hinwegsetzen ${ }^{16}$, doch kann er einen Dissens innerhalb der Fachwelt mit der eigenen Überzeugung entscheiden ${ }^{17}$. Die Richter im Contergan-Verfahren durften folglich aufgrund der signifikanten Übereinstimmung der Kausalverläufe und anderer Indizien, die theoretischen Zweifel unberücksichtigt lassen und die Kausalität des Produktfehlers für die Körperschäden bei den Neugeborenen bejahen $^{18}$.

So wurde auch im Holzschutzmittelfall 1992 entschieden. ${ }^{19}$ Viele Kunden hatten das Holzschutzmittel der Marke „Xyladecor“ genutzt. 2100 Personen klagten daraufhin über unspezifische Symptome, wie Kopfschmerzen, Mattigkeit und Übelkeit. Ein echter wissenschaftlicher Nachweis, dass diese inhomogenen Krankheitsbilder, die unter dem Begriff „Holzschutzmittelsyndrom“ bekannt wurden, direkt auf die Giftstoffe des Mittels im Niedrigdosisbereich zurückzuführen waren, gelangt nicht, ist aber auch nicht notwendig. Die Feststellung der Kausalität verlange keine absolut

${ }^{13}$ Schönke/Schröder/Sternberg-Lieben, $§ \quad 15$ Rn. 223b; Kudlich/Oglakcioglu, Wirtschaftsstrafrecht, 2011, S. 47, Rn. 128.

${ }^{14}$ BGH NJW 1990, S. 2562; Kuhlen (Fn. 1), S. 72.

${ }^{15}$ LG Aachen, JZ 1971, 511.

${ }^{16}$ BGHSt 29, 18, 21; Kuhlen (Fn. 1), S. 67; Karlsruher Kommentar StPO/Schoreit, 6. Aufl. München 2008, § 261 StPO Rn. 46.

${ }^{17}$ Kuhlen, a.a.O., a.A. Kaufmann, JZ 1971, S 573 f.: Die Existenz eines Kausalgesetzes müsse als Teil des objektiven Tatbestands objektiv gewiss sein.

${ }^{18}$ Tiedemann, Wirtschaftsstrafrecht AT, 3. Auflage, Köln 2010, Rn. 171.

${ }^{19}$ BGH, StV 1997, S. 124 ff. Kritisch dazu Schulz, Strafrechtliche Produkthaftung bei Holzschutzmitteln, Zeitschrift für Umweltrecht 1994, S. 26, 30 ff. 
unanzweifelbare Gewissheit, so der BGH. Es genüge, dass ernsthafte Zweifel nicht bestehen. Damit wird freilich ein wenig in die Trickkiste gegriffen, indem Beweisschwierigkeiten dadurch ausgeräumt werden, dass man die Anforderungen an den Nachweis zurückschraubt.

Die Kausalitätsvoraussetzung ist demnach zwar ein Strafbarkeitshindernis, aber kein unüberwindbares.

\section{Feststellung der objektiven Sorgfaltspflichtverletzung}

Bei der Frage, wie die ordnungsgemäße Sorgfalt eines Produzenten aussieht und ob etwa bei der Herstellung oder dem Inverkehrbringen eines Produkts eine Sorgfaltspflichtverletzung vorliegt, stößt der Strafrechtler zunächst auf das Problem, dass das Unternehmen selbst strafrechtlich nicht zur Verantwortung gezogen werden $\mathrm{kann}^{20}$. Anders als im Zivilrecht, wo das Unternehmen als juristische Person zum Schadensersatz in Anspruch genommen werden kann, knüpft das StGB ausgehend vom verfassungsrechtlich in Art. 1 Abs. 1 GG verankerten Schuldgrundsatz an die persönliche Haftung für das Fehlverhalten des Einzelnen an, so dass sich die strafrechtliche Verantwortung auf die Verantwortung der einzelnen Mitarbeiter verlagert ${ }^{21}$. Eine Verbandssanktion in Form einer Geldbuße kennt allein das Ordnungswidrigkeitenrecht in $\S 30 \mathrm{OWiG}^{22}$. Wenn es aber um die Strafbarkeit des Produktfehlers geht, ist zunächst zu klären, wer innerhalb des arbeitsteilig organisierten Organismus eines Unternehmens zur Verantwortung gezogen werden kann.

\section{a) Frage nach dem Verantwortlichen innerhalb der Unternehmenshierarchie}

Beginnt man dabei mit dem primären Sorgfaltspflichtverstoß, fragt man mithin, wer die letzte Ursache für den Erfolg gesetzt hat, so wird diese Fragestellung kaum viel Klarheit bringen. Ein Letztverursacher ist auf allen Ebenen des Unternehmens denkbar, sei es ein Arbeiter, der eine letzte Sicherungsschraube vergisst, sei es der Beschluss des Vorstandsvorsitzenden, eine erkannt mangelhafte Ware weiter zu verkaufen. Da sich damit Überschneidungen mit den Personenbereichen ergeben, die in der Leitung und Aufsicht als mittelbar Verpflichtete ihre Organisationspflichten verletzt haben können, ist diese Differenzierung

\footnotetext{
${ }^{20}$ Schmidt-Salzer (Fn. 6), NJW 1988, S. 1938; ders., (Fn. 1) NJW 1990, S. 2967.

${ }^{21}$ Schmidt-Salzer (Fn. 6), NJW 1988, S. 1938.

${ }^{22}$ Schmidt-Salzer (Fn. 6), NJW 1988, S. 1938; Schönke/Schröder/Sternberg-Lieben, § 15 Rn. $223 \mathrm{e}$.
} 
zwischen primärem und sekundärem Verantwortlichen als gekünstelt zu kritisieren.

Geeigneter Anknüpfungspunkt für einen Sorgfaltspflichtverstoß ist die Organisationsstruktur des Unternehmens: aus dessen Ressortverteilung und dem Organisationsplan können diejenigen Mitarbeiter bestimmt werden, die für eine jeweilige Teilerfüllung verantwortlich $\operatorname{sind}^{23}$.

Dabei entsteht zwischen den Teilverantwortlichkeiten kein leerer verantwortungsloser Raum ${ }^{24}$. Die strafrechtliche Produktverantwortlichkeit lässt sich als ein Mosaik von Einzelverantwortlichkeiten der Mitarbeiter eines Unternehmens sehen, die bezogen auf das Unternehmen als Ganzes das Bild eines sorgfältig handelnden Gesamtunternehmens ergeben ${ }^{25}$. Die innerbetriebliche Zuständigkeitsverteilung bestimmt also, wer strafrechtlich verantwortlich sein könnte.

An diese Feststellung schließt sich sofort die Kritik an, dass sich Unternehmensleitung und das mittlere Management gegenseitig die Verantwortung zuschieben könnten ${ }^{26}$ oder sogar ganz ihre strafrechtlich an sich gegebene Verantwortung auf die untersten Instanzen der Unternehmenshierarchie verlegen könnten und damit „die ,Organisierung der Verantwortlichkeit' in die ,organisierte Unverantwortlichkeit' umzuschlagen (drohte) ${ }^{627}$.

Dieser Einwand überzeugt aus zwei Gründen nicht. So ist bei lückenhafter oder bewusst inadäquater Aufgabenverteilung im Betrieb stets auch der zu verurteilen, der die Verantwortung kraft tatsächlicher Funktionsübernahme oder kraft Zuordnung nach der Natur der Sache trägt ${ }^{28}$. Unklare Kompetenzen schützen also die Mitarbeiter nicht.

${ }^{23}$ Schmidt-Salzer (Fn. 6), NJW 1988, S. 1940; ders., (Fn. 1), NJW 1990, S. 2968; Satzger/Schmitt/Widmaier/Momsen, Köln 2009, §§ 15,16 Rn. 71.

${ }^{24}$ Schmidt-Salzer, Zivilrechtliche und strafrechtliche Produktverantwortung, JA 1988, S. 474; ders., (Fn. 1), NJW 1990, S. 2968.

${ }^{25}$ Schönke/Schröder/Sternberg-Lieben, § 15 Rn. 223a.

${ }^{26}$ Schünemann, Unternehmenskriminalität und Strafrecht, Köln, Berlin, Bonn, München 1979, S. 44.

${ }^{27}$ Schünemann, Strafrechtsdogmatische und kriminalpolitische Grundfragen der Unternehmenskriminalität, wistra 1982, S. 42.

${ }^{28}$ Schmidt-Salzer (Fn. 6), NJW 1988, S. 1940; ders. (Fn.1), NJW 1990, S. 2970; Brendl, Straftatrisiko bei Schutzgesetzen, in: ders., Produkt- und Produktenhaftung, Gruppe 11, S. $74 \mathrm{f}$. 
Zum zweiten führt die strafrechtliche Arbeitsteilung nicht zu einer bloßen strafrechtlichen Verantwortungsteilung ${ }^{29}$. Auch bei einer zulässigen Pflichtenübertragung bleibt bei dem Vorgesetzten eine Mitverantwortung in Form von Kontroll- und Überwachungspflichten bestehen. So werden sie etwa nicht von der Pflicht befreit, für die richtige Auswahl, die präzise Anweisung und Überwachung derjenigen Sorge zu tragen, an die sie eigene Aufgabenstellungen delegiert haben ${ }^{30}$. Da sich damit verschiedene Verantwortlichkeitsbereiche überlagern und ergänzen ${ }^{31}$, führt die Arbeitsteilung $\mathrm{zu}$ einer Vervielfachung der individuellen Verantwortlichkeiten ${ }^{32}$.

Hier ist das Ergebnis festzuhalten, dass man grundsätzlich für den eigenen individuellen Verantwortungsbereich haftet, der sich in erster Linie - aber nicht ausschließlich - aus dem Struktur- und Organisationsplan ergibt.

Daneben trifft die Mitglieder der Geschäftsleitung außerhalb ihres individuellen Haftungsbereichs die sog. Generalverantwortung, die im Lederspray-Urteil auch vom BGH anerkannt wurde ${ }^{33}$. Es ging um giftige Gase in Schuhpflege-Sprays der bekannten Marke „Erdal Rex“, die zu gefährlichen Lungenerkrankungen geführt hatten. Nach diesem Prinzip haftet jedes Mitglied des Kollegialorgans, wenn dieses sorgfaltswidrig gehandelt hat ${ }^{34}$, und zwar in zwei Fällen, in denen das Unternehmen als Ganzes betroffen ist.

- So bleibt die Geschäftsführung für die ordnungsgemäße Organisation des Betriebes als der Grundpflicht überhaupt haftbar ${ }^{35}$.

- Des Weiteren fällt eine zunächst übertragene Verantwortung in Krisen- und Ausnahmesituationen, etwa bei der Entscheidung über eine Rückrufaktion, auf die Geschäftsleitung zurück ${ }^{36}$.

\footnotetext{
${ }^{29}$ Schmidt-Salzer (Fn. 6), NJW 1988, S. 1937.

${ }^{30}$ Schmidt-Salzer (Fn. 6), NJW 1988, S. 1941; ders. (Fn. 1), NJW 1990, S. 2970; allgemein dazu Schumann, Strafrechtliches Handlungsunrecht und das Prinzip der Selbstverantwortung der Anderen, Tübingen 1986, S. 26 f.; Schönke/Schröder/Stree, § 13 Rn. 26; BGHSt 19, 286, 288 f.

${ }^{31}$ Vgl. Schmidt-Salzer (Fn. 6), NJW 1988, S. 1942.

${ }^{32}$ Schmidt-Salzer (Fn. 24), JA 1988, S. 474; Brendl (Fn. 28), 11/74 d.

${ }^{33}$ BGH NJW 1990, S. 2564 f.; Schmidt-Salzer (Fn. 6), NJW 1988, S. 1939 f.; ders. (Fn. 1), NJW 1990, S. 2967.

${ }^{34}$ Schönke/Schröder/Sternberg-Lieben, § 15 Rn. 223b.

${ }^{35}$ Schmidt-Salzer (Fn. 6), NJW 1988, S. 1941; Schünemann (Fn. 26), S. 107.

${ }^{36}$ BGH NJW 1990, S. 2565; Schmidt-Salzer (Fn. 1), NJW 1990, S. 2968.
} 
Mit dem hier vorgestellten strafrechtlichen Instrumentarium scheint kein Produktfehler denkbar, für den sich nicht auch ein strafrechtlich Verantwortlicher finden ließe.

\section{b) Inhalt der Sorgfaltspflicht}

Der Sorgfaltsmaßstab ist im Strafrecht zunächst mit dem im Zivilrecht identisch $^{37}$. Die Festlegung der einzelnen Sorgfaltspflichten bestimmt sich auch hier nach dem Durchschnitt, also nach dem Verhalten, das ein gewissenhafter Mensch des Verkehrskreises, dem der Handelnde angehört, in einer bestimmten Situation anzuwenden pflegt $^{38}$. Konkret ist demnach danach zu fragen, wie sorgfältig sich der Hersteller eines bestimmten Wirtschaftszweiges bei der Herstellung von Produkten verhält.

Der konkrete Inhalt einer Sorgfaltspflicht, seien es nun generelle Organisationspflichten, Warnungs-, Überwachungs- oder Auswahlpflichten, lässt sich nur am Einzelfall bestimmen. Generell kann aber dem grundsätzlichen Verbot der Rechtsordnung, Gefahren zu schaffen, die andere schädigen (neminem laedere) ${ }^{39}$, die allgemeine Pflicht für einen Produzenten entnommen werden, seine Abnehmer vor Gefährdungen durch seine Waren zu bewahren. Es sollte aber angenommen werden, dass ein Produzent davon ausgehen darf, dass er pflichtgemäß handelt, sofern eine behördliche Zulassung vorliegt ${ }^{40}$. Freilich wird dieser auch durch die behördliche Zulassung seines Produkts nicht von seinen Kontroll-, Beobachtungs- und Rückrufpflichten entbunden ${ }^{41}$. Dabei wird an seine Sorgfaltsanforderungen ein umso höherer Maßstab anzulegen sein, je höher die Gefährlichkeit eines Fehlers für den Produktbenutzer ist ${ }^{42}$.

\section{c) Haftung für Unterlassen}

Eine besondere Problematik ergibt sich bei Warn- und Rückrufpflichten. Da die Verantwortlichen im Unternehmen hier für eine unterlassene Reaktion auf erkennbare Gefahrenquellen strafrechtlich belangt werden sollen und es folglich um die Unterlassensstrafbarkeit geht, muss zunächst eine Garantenstellung zu bejahen $\operatorname{sein}^{43}$.

\footnotetext{
${ }^{37}$ Kuhlen (Fn. 1), S. 172.

${ }^{38}$ Schönke/Schröder/Sternberg-Lieben, § 15 Rn. 19; Kuhlen (Fn. 1), S. 84.

${ }^{39}$ BGH NJW 1990, S. 2562 f.; vgl. das allgemeine Schädigungsverbot alterum non laedere, LG München II ES, S. 330.

40 Achenbach/Ransiek/Kuhlen, Wirtschaftsstrafrecht, 3. Aufl., Heidelberg 2012, Rn. 36.

${ }^{41}$ LK-Vogel, 12. Auflage, Berlin 2007, § 15 Rn. 280.

${ }^{42}$ LG Aachen, JZ 1971, S. 516; LG München II (Fn. 39).

${ }^{43}$ Kudlich/Oglakcioglu, Wirtschaftsstrafrecht, Heidelberg 2011, Rn. 142.
} 
aa) $\mathrm{Ob}$ eine sog. „Geschäftsherrenhaftung“ besteht, wonach der Betriebsinhaber bzw. die Geschäftsführer generell verpflichtet sind, betriebsbezogene Straftaten der anderen, frei verantwortlichen handelnden Mitarbeiter zu verhindern, ist höchst umstritten ${ }^{44}$. Die wohl überwiegende Auffassung will eine solche Garantenstellung nur für gefährliche Betriebe annehmen ${ }^{45}$.

bb) Es bleibt die Herleitung einer Garantenstellung aus einem pflichtwidrigen Vorverhalten (der sog. Ingerenz). Dies gelingt ohne Probleme, soweit bereits in dem Inverkehrbringen des fehlerhaften Produktes an sich ein Sorgfaltsverstoß $\mathrm{zu}$ sehen ist, wenn die Vertriebsfreigabe z.B. verfrüht erfolgte.

Schwieriger zu lösen sind jene Fälle, bei denen die Gefährlichkeit des Produktes erst später deutlich wird. Z.T. wird mit dem Argument, das Produkt habe den Herrschaftsbereich des Herstellers verlassen, eine Garantenstellung abgelehnt ${ }^{46}$. Dem hat der BGH im Erdal-Rex-Urteil überzeugend widersprochen. So habe der Produzent im Rückruf durchaus ein Mittel in der Hand, auf die fehlerhaften Produkte Einfluss zu nehmen. Auch besitze allein er den nötigen Überblick und die Sachkenntnis, um das Richtige zu veranlassen ${ }^{47}$. Demnach obliegt demjenigen, der mangelhafte Produkte in den Verkehr bringt und Gefahren für die Verbraucher herbeiführt, eine Garantenpflicht aus Ingerenz ${ }^{48}$.

Denkbar wäre auch, diese Garantenstellung aus der Produktbeobachtungspflicht, die im Zivilrecht uneingeschränkt anerkannt ist und ebenfalls Warn- und Rückrufpflichten begründet, herzuleiten ${ }^{49}$.

cc) Wann eine solche Pflicht in concreto besteht, hängt wiederum von den Umständen des Einzelfalles ab. Allgemein wird ein Unternehmen nicht zuwarten dürfen, bis über die Gefährlichkeit der Produkte sichere Klarheit herrscht, vielmehr verlangen begründete Verdachtsmomente eine Reaktion ${ }^{50}$, wobei bei einer Häufigkeit festgestellter Schäden oder der Irreversibilität -

${ }^{44}$ Vgl. Lackner/Kühl StGB, 27. Aufl., § 13 Rn. 13 m.w.N.; sowie Tiedemann, Wirtschaftsstrafrecht AT, 3. Auflage, Köln 2010, Rn. 181 ; ablehnend Schünemann (Fn. 27), wistra 1982, S. 43 m.w.N.

${ }^{45}$ Tiedemann, Wirtschaftsstrafrecht - Einführung und Übersicht, JuS 1989, S. 697.

${ }^{46}$ Schünemann (Fn. 27), wistra 1982, S. 44.

${ }^{47}$ BGH NJW 1990, S. 2564.

${ }^{48}$ BGH NJW 1990, S. 2563.

${ }^{49}$ Tiedemann, Wirtschaftsstrafrecht, 2. Aufl. Köln u.a. 2008, Rn. 531; Eidam (Fn. 1), Rn. 2421 ff.; offengelassen von BGH NJW 1990, S. 2562.

${ }^{50}$ BGH NJW 1990, S. 2564; Schönke/Schröder//Sternberg-Lieben, § 15 Rn. 223d; LG Aachen, JZ 1971, S. 515. 
wie im Contergan-Fall - schon die entfernte Möglichkeit, der Verdacht sei richtig, genügen wird ${ }^{51}$.

\section{Einschränkung der Pflichtwidrigkeit}

Neben der Feststellung einer Sorgfaltspflichtverletzung setzt die Fahrlässigkeitsstrafbarkeit den sog. Pflichtwidrigkeitszusammenhang voraus. Im Bereich der Produktfehlerhaftung könnte diese Relevanzprüfung unter dem Aspekt des erlaubten Risikos scheitern.

Zunächst lässt sich auch bei einwandfreier Massenproduktion technisch nicht gewährleisten, dass wirklich jedes Produkt fehlerfrei und ungefährlich bleibt. Das Risiko solcher Ausreißer ist unvermeidbar und wird als erlaubtes Risiko hingenommen, soweit ein anerkanntes Stichprobenverfahren existiert $^{52}$.

Daneben genügt es, dass die Produkte - sofern von den Verbrauchern nicht mehr erwartet wird - eine gewisse Grundsicherheit aufweisen: Man darf nicht nur völlig ,idiotensichere“ Produkte auf den Markt bringen ${ }^{53}$.

So wird ein Restrisiko meist von der allgemeinen Verkehrserwartung toleriert (es wird damit zum erlaubten Risiko), weil etwa ein höchst sicheres Auto für Jedermann mit Antiblockiersystem, Airbag, Allradantrieb, Abstandswarngerät etc. zwar technisch realisierbar, aber ökonomisch nicht vertretbar wäre.

Der zweite Unterfall des erlaubten Risikos wird näher durch den Vertrauensgrundsatz bestimmt. Allgemein besagt dieser, dass man, solange man sich selbst sorgfältig verhält, auf das sorgfältige Verhalten der anderen vertrauen darf. Dieser im Straßenverkehrsrecht entwickelte Grundsatz gilt auch in der Warenproduktion. Der Produzent darf auf den ordnungsgemäßen Gebrauch seiner Produkte vertrauen ${ }^{54}$. Er hat zwar vor Gefahrpotentialen in verständlicher Weise aufzuklären ${ }^{55}$ und muss ein unbewusstes Verbraucherfehlverhalten nach Möglichkeiten mitberücksichtigen ${ }^{56}$, doch

\footnotetext{
${ }^{51}$ LG Aachen, JZ 1971, S. 516.

${ }^{52}$ LG München II, ES, S. 320.

${ }^{53}$ Schmidt-Salzer (Fn. 12), S. 237.

${ }^{54}$ Schönke/Schröder/Sternberg-Lieben, § 15 Rn. 223f; Schumann (Fn. 29), S. 23; Kuhlen (Fn. 1), S. $139 \mathrm{f}$.

${ }^{55}$ Schönke/Schröder/Sternberg-Lieben $§ 15$ Rn. 223f; LK-Vogel, 12. Auflage, Berlin 2007, § 15 Rn. 279.

${ }^{56}$ Vgl. § 3 I ProdHaftG: Das Produkt muss die Sicherheit für einen Gebrauch bieten, „mit dem billigerweise gerechnet werden kann“. Weitergehend Tiedemann (Fn. 49), Rn. 532.
} 
schließt eine gezielt zweckentfremdete Benutzung seine Verantwortlichkeit aus ${ }^{57}$.

So wäre in dem bekannten „Sniffing“-Fall, bei dem Jugendliche sich an einem technischen Lösungsmittel (mit tödlichem Ausgang) berauschten, der Hersteller nicht strafbar, weil dieses Mittel nur für den gewerblichen Gebrauch durch Fachleute bestimmt war und ein derartig bewusst bestimmungswidriger Gebrauch nicht vorauszusehen war $^{58}$.

Der Vertrauensgrundsatz entlastet unter Umständen den Unternehmer auch im Innenverhältnis seiner von ihm beherrschten Organisation. Er darf darauf vertrauen, dass seine Mitarbeiter besonnen handeln, sofern er diese nach einer gut bedachten Auswahl eingestellt hat und durch stichprobenhafte Überwachung kontrolliert ${ }^{59}$.

\section{Die Zumutbarkeit normgemäßen Verhaltens}

Für den Schuldvorwurf ist nun ein subjektiver Maßstab heranzuziehen. Dabei sind die Kenntnisse und Fähigkeiten der Einzelnen maßgelblich: konnte der Täter nach seinem individuellen Vermögen seine Anforderungen erkennen und erfüllen? Der objektiv-generelle Ansatz des Zivilrechts wird also durch einen subjektiv-konkreten ergänzt. Daneben ergeben sich auf Schuldebene interessante Probleme, soweit es um die normativ-wertende Frage der Zumutbarkeit pflichtgemäßen Verhaltens geht ${ }^{60}$.

Die Zumutbarkeit spielt auf zwei Ebenen eine Rolle. Soweit das Unternehmen als Ganzes betroffen ist, geht es in der Regel um die Frage, ob eine öffentliche Warnung oder ein Rückruf unter dem Aspekt der Unzumutbarkeit unterlassen werden durfte, was bei den Unterlassungsdelikten zum Ausschluss der Tatbestandsmäßigkeit führte.

Bezüglich der einzelnen Verantwortlichen stellt sich die Frage, ob nicht drohende Nachteile für die eigene Person eine Fahrlässigkeitsstrafbarkeit entfallen lassen.

a) Was das Unternehmen anbelangt, so ist $\mathrm{zu}$ konzidieren, dass Rückrufe und Warnhinweise in der Öffentlichkeit mit einem Imageverlust verbunden sein können und gegebenenfalls sogar zu Gewinneinbußen führen.

\footnotetext{
${ }^{57}$ BGH NJW 1990, S. 2562; Kuhlen (Fn. 1), S. 124.

${ }^{58}$ Siehe BGH (6. Zivilsenat), NJW 1981, S. 2514, 2516 f.

59 MüKo-StGB/Duttge, 2. Auflage, München 2011, § 15 Rn. 146.

${ }^{60}$ Unzumutbarkeit ist nach h.M. ein schuldbegrenzendes Kriterium der subjektiven Sorgfaltspflicht.
} 
Allerdings ist für die Prüfung der Unzumutbarkeit eine Interessenabwägung maßgebend, bei der die drohenden Nachteile für den pflichtgemäß handelnden Unternehmer auf der einen Seite mit der Wahrscheinlichkeit und Schwere eine drohenden Rechtsgutsverletzung auf der anderen Seite abzuwägen $\operatorname{sind}^{61}$. Dabei gebührt dem Schutz des Verbrauchers vor Gesundheitsschäden stets der Vorrang vor wirtschaftlichen Überlegungen $^{62}$. Allenfalls bei geringfügigen Nachteilen bei den Konsumenten und existenzgefährdenden Folgen einer Rückrufaktion könnte ein Einschreiten durch ein Unternehmen unzumutbar $\operatorname{sein}^{63}$.

b) Was die einzelnen Arbeitnehmer anbelangt, so erkennt man bereits an der Auffassung der h.M., nicht einmal die Gefahr einer Strafverfolgung dürfe jemanden vom pflichtgemäßen Verhalten abhalten ${ }^{64}$, dass sehr hohe Anforderungen an die Zumutbarkeit des Tätigwerdens gestellt werden.

So sollen persönliche Schwierigkeiten im Betrieb und sogar berufliche Nachteile nicht genügen, um jemanden vom gebotenen Handeln abzuhalten $^{65}$.

Bei einem falschen Mehrheitsbeschluss innerhalb eines Kollegialorgans wird der Einzelne nicht durch bloßes Dagegenvotieren befreit, noch weniger darf er diese eindeutig sachwidrige Entscheidung mittragen. Vielmehr hat er auf einen richtigen Beschluss $\mathrm{zu}$ drängen, die Sache an die Unternehmensleitung weiterzugeben und unter Umständen - etwa bei der unmittelbaren Gefahr erheblicher Schäden - sogar an die Öffentlichkeit zu gehen $^{66}$. Allerdings soll man nicht auf eigene Faust gegen den ausdrücklichen Willen der Geschäftsführung die erforderlichen Maßnahmen ergreifen müssen ${ }^{67}$.

Auch sind untergeordnete Mitarbeiter nicht verpflichtet, die von Ihnen befürwortete Rückrufmaßnahme gegen den Willen der Geschäftsführung durchzusetzen - mangels Entscheidungskompetenz wären sie dazu auch gar nicht in der Lage. Vielmehr haben sie mit der Information der Geschäftsführung alles Ihnen Mögliche und Zumutbare getan, um den

${ }^{61}$ Schönke/Schröder/Lenckner/Sternberg-Lieben, $\quad$ Vorbem. $\$ \S 32$ ff. $\quad$ Rn. 126; Achenbach/Ransiek/Kuhlen, Wirtschaftsstrafrecht, 3. Aufl., Heidelberg 2012, Rn. 28.

${ }^{62}$ BGH NJW 1990, S. 2564; LG Aachen, JZ 1971, S. 515; Schmidt-Salzer (Fn. 12), S. 210 f.

${ }^{63}$ BGH NJW 1990, S. 2564.

${ }^{64}$ Vgl. nur BGHSt 11, 353, 355.

${ }^{65}$ LG Aachen, JZ 1971, S. 519.

${ }^{66}$ BGH NStE Nr. 5 zu $\S 223$; Momsen/Grützner/Voigtel, Wirtschaftsstrafrecht, München 2013, Rn. 64.

${ }^{67}$ BGH NStE Nr. 5 zu $§ 223$. 
Eintritt weiterer Schäden abzuwenden. Damit kann sich das Risiko der strafrechtlichen Produkthaftung durch gewissenhafte Information an die übergeordneten Stellen "verschieben" und letztlich diejenigen treffen kann, die die letzte Entscheidungsgewalt in einem Unternehmen ausüben.

Das macht auch verständlich, warum letztlich die strafrechtliche Produkthaftung mehr gefürchtet ist als die zivilrechtliche. Sie bildet ein wesentliches Element der Fragen, die mit den Stichworten criminal compliance in der modernen Strafrechtsdiskussion umschrieben werden ${ }^{68}$.

68 S. nur Rotsch, Criminal Compliance, ZIS 2010, 614 ff. 


\section{BİBLİOGRAFİE}

Vgl. Schmidt-Salzer, Strafrechtliche Produktverantwortung, Das Lederspray-Urteil des BGH, NJW 1990.

Kuhlen, Fragen einer strafrechtlichen Produkthaftung, Heidelberg 1989.

Momsen/Grützner/Voigtel, Wirtschaftsstrafrecht, München 2013.

Schmidt-Salzer, Entscheidungssammlung, Produkthaftung, Band IV, München 1982.

Schmidt-Salzer, Strafrechtliche Produktverantwortung, NJW 1988. Achenbach/Ransiek/Kuhlen, Wirtschaftsstrafrecht, 3. Aufl., Heidelberg 2012.

Tiedemann, Fragen einer strafrechtlichen Produkthaftung, NJW 1990.

Schmidt-Salzer, Produkthaftung, Band I: Strafrecht, 2. Aufl., Heidelberg 1988.

Kudlich/Oglakcioglu, Wirtschaftsstrafrecht, Heidelberg 2011.

Karlsruher Kommentar StPO/Schoreit, 6. Aufl. München 2008.

Strafrechtliche Produkthaftung bei Holzschutzmitteln, Zeitschrift für Umweltrecht 1994.

Schmidt-Salzer, Zivilrechtliche und strafrechtliche Produktverantwortung, JA 1988, S. 474; ders., (Fn. 1), NJW 1990. Schünemann, Unternehmenskriminalität und Strafrecht, Köln, Berlin, Bonn, München 1979.

Schünemann, Strafrechtsdogmatische und kriminalpolitische Grundfragen der Unternehmenskriminalität, wistra 1982.

Brendl, Straftatrisiko bei Schutzgesetzen, in: ders., Produkt- und Produktenhaftung, Gruppe 11.

Strafrechtliches Handlungsunrecht und das Prinzip der Selbstverantwortung der Anderen, Tübingen 1986.

Achenbach/Ransiek/Kuhlen, Wirtschaftsstrafrecht, 3. Aufl., Heidelberg 2012.

LK-Vogel, 12. Auflage, Berlin 2007.

Tiedemann, Wirtschaftsstrafrecht AT, 3. Auflage, Köln 2010.

Tiedemann, Wirtschaftsstrafrecht - Einführung und Übersicht, JuS 1989.

Tiedemann, Wirtschaftsstrafrecht, 2. Aufl. Köln u.a. 2008.

MüKo-StGB/Duttge, 2. Auflage, München 2011. 
